\title{
Buckling analysis of tape springs using a rod model with flexible cross-sections
}

\author{
Stéphane Bourgeois ${ }^{\mathrm{a}, \mathrm{b} *}$, Bruno Cochelin ${ }^{\mathrm{a}, \mathrm{b}}$, François Guinot ${ }^{\mathrm{c}}$ and Elia Picault ${ }^{\mathrm{a}}$ \\ ${ }^{a}$ Laboratoire de Mécanique et d'Acoustique - UPR CNRS 7051, 31 chemin Joseph-Aiguier, F-13402 \\ Marseille Cedex 20, France; ${ }^{b}$ Ecole Centrale Marseille, Technopôle de Château-Gombert, 38 rue Joliot \\ Curie, F-13451 Marseille Cedex 20, France; ${ }^{c}$ Thales Alenia Space, 100 bd du midi - B.P. 99, F-06156 \\ Cannes La Bocca Cedex, France
}

\begin{abstract}
This work is devoted to the study of tape springs behaviour. We focus on the instabilities that lead to the creation of localised folds, due to the flattening of the cross-section. Depending on the kind of loading, the folds can move along the tape, split or disappear. Using an extended four parameters rod model with flexible cross-sections, we study several modes of instabilities (number and localisation of folds) for such structures submitted to an overall compression or bending.

On s'intéresse à la modélisation du comportement des mètres rubans, qui ont la particularité de développer des pliages localisés dus à un aplatissement par flambage de la section. Suivant les scénarios de chargement, les zones de pliages peuvent se déplacer le long du ruban, se dupliquer ou disparaître. S'appuyant sur un modèle original de poutre à section flexible à quatre paramètres cinématiques, on étudie les différents modes d'instabilités (nombre et localisation des pliages) de ces structures soumises à des sollicitations de compression et de flexion dans le plan.
\end{abstract}

Keywords: tape springs; folding; buckling; rod model

Mots-clés: mètres rubans; pliage; flambement; modèle de poutre

\section{Introduction}

A crucial point for satellite manufacturers is to develop ingenious compact systems that can have an autonomous deployment during transfer orbit. In this framework, deployable structures composed of folded tape springs can offer an interesting alternative to articulated rigid mechanisms (Santer \& Pellegrino, 2008; Seffen \& Pellegrino, 1999). They may lead to more compact systems and may increase fiability by suppressing hinges, bolts and motorisations. However, the deployment needs to be perfectly controlled and the modelling of tape springs behaviour becomes a challenging issue.

In its free configuration, a tape spring can be assimilated to a straight rod with an open and thin-walled cross-section of constant transverse curvature. As shown in Figure 1, when an overall bending is applied, the tape behaves at first as a classical beam (with a more or less uniform bending curvature) but there is suddenly the creation of a localised fold (Seffen, 2001; Seffen \& Pellegrino, 1999), indicating snap-through buckling. These folds occur, thanks

*Corresponding author. Email: stephane.bourgeois@centrale-marseille.fr

ISSN 1779-7179 print/ISSN $1958-5829$ online

(C) 2012 Taylor \& Francis

http://dx.doi.org/10.1080/17797179.2012.714848

http://www.tandfonline.com 


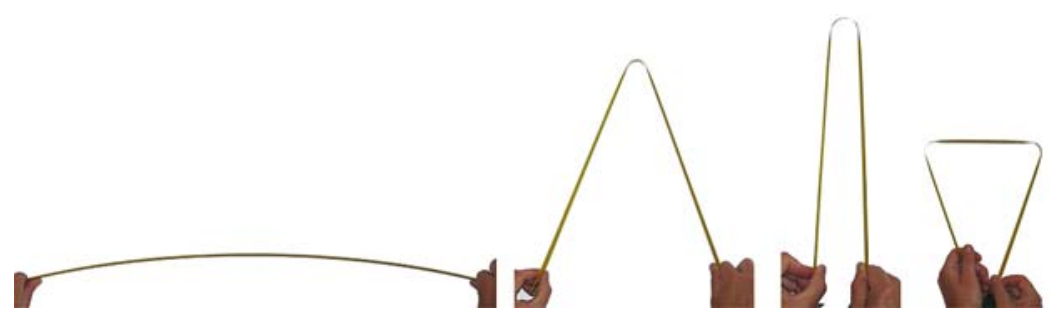

Figure 1. Folding of a measuring tape.

to the flexibility of the cross-section, which becomes flat in some localised areas to decrease the whole strain energy. It leads to concentrate the deformation in the region of the fold, whereas the rest of the tape recovers its undeformed shape. Playing with a measuring tape, one can easily experience the creation of one or several folds, the migration of a fold along the tape, the splitting of a single fold into two or the merging of two folds into one.

To simulate these buckling phenomena, the first approach consists of using a non-linear elastic shell model in the framework of large displacements and large rotations. This can be performed by using a commercial finite element software package (Hoffait, Bruls, Granville, Cugnon, \& Kerschen, 2009; Seffen, You, \& Pellegrino, 2000; Soykasap, 2007; Walker \& Aglietti, 2007). This approach, which can be used for any kind of loading, provides accurate results but leads to heavy and hard to drive computations. A more suitable approach consists of taking into account the specificities of the tape springs behaviour. For instance, some authors (Seffen \& Pellegrino, 1999) consider the tape in its folded configuration as an assembly of two rigid bars and a non-linear spiral spring accounting for the bending stiffness of the fold. The rigid bars are of variable length to allow the migration of the fold along the tape. It leads to a non-linear system with two degrees of freedom, which is successfully used to model some experiments of dynamic deployment. Unfortunately, this method requires the introduction of the folds (number and localisation) ab initio and is not able to account for the creation of new folds.

Recently, an intermediate approach has been proposed in which the tape spring is considered as a rod with highly flexible cross-sections (Guinot, Bourgeois, Cochelin, \& Blanchard, 2012). This approach appears to be more general than the above mentioned discrete model and easier to handle than the full non-linear shell model. Many works have been published on beam models with deformable cross-sections (see e.g. Gonçalves, Ritto-Corrêa, \& Camotim, 2010; Pimenta \& Campello, 2003; Simo \& Vu-Quoc, 1991; Vlassov, 1962; Zivkovic, Kojic, Slavkovic, \& Grujovic, 2001). The main idea is always to incorporate shape functions to describe the deformation of the cross-section. The method proposed in Guinot et al. (2012) follows this idea. Starting from a non-linear shell model in the framework of large displacements, large rotations and dynamics, the main novelty resides in the use of an elastica kinematics to account for the large changes of the cross-section shape with very few parameters. At last, it leads to a planar rod model with only four kinematic parameters.

The present work aims at showing that this quite simple model is able to handle complex scenarios of buckling, taking into account the creation and disappearance of folds depending on the loading path. The key points of the general model presented in Guinot et al. (2012) and its implementation using an energetic approach are first recalled for the restricted case of tape springs with a circular cross-section. In a second part, several applications examples illustrate the ability of the model to face with complex scenarios of buckling, involving the creation, the duplication and the migration of folds along the tape. 


\section{The rod model}

\subsection{Kinematic description and basic assumptions}

Starting from a shell model, a tape spring is regarded as a rod described in its initial free configuration by a straight rod line and a planar circular cross-section curve, as shown in Figure 2. The fixed orthonormal frame $\left(O, \mathbf{e}_{\mathbf{1}}, \mathbf{e}_{\mathbf{2}}, \mathbf{e}_{\mathbf{3}}\right)$ is chosen such that the axis $\left(O, \mathbf{e}_{\mathbf{1}}\right)$ contains the rod line and that the plane $\left(O, \mathbf{e}_{1}, \mathbf{e}_{3}\right)$ is the plane of symmetry of the crosssection curve.

We introduce a curvilinear coordinate system $\left(s_{1}, s_{2}\right) \in[0, L] \times[-a / 2, a / 2]$ to map the geometry of the tape, with $L$ the initial length of the rod line and $a$ the initial length of the cross-section curve. In the deformed configuration, the position of a material point $M$ is given by:

$$
\mathbf{O M}\left(s_{1}, s_{2}\right)=\mathbf{O G}\left(s_{1}\right)+\mathbf{G M}\left(s_{1}, s_{2}\right),
$$

where $G$ is the point of the cross-section on the rod line. The rod model kinematics presented in Guinot et al. (2012) relies on four assumptions:

(i) the cross-section curve remains in a plane after deformation;

(ii) the cross-section plane is orthogonal to the tangent vector of the rod line in the deformed configuration;

(iii) the cross-section curve is considered inextensible and

(iv) the shape of the tape remains symmetric with respect to the plane $\left(O, \mathbf{e}_{1}, \mathbf{e}_{3}\right)$.

The two first assumptions are the classical hypotheses used in the Euler-Bernoulli beam theory. In accordance with the assumption (iv), the motion of the rod line is restrained to the plane $\left(O, \mathbf{e}_{1}, \mathbf{e}_{3}\right)$ : the displacement of a point $G$ on the rod line is then given by the two components $u_{1}\left(s_{1}\right)$ and $u_{3}\left(s_{1}\right)$, and the rotation of the cross-section plane $\theta\left(s_{1}\right)$ is around the axis $\mathbf{e}_{2}$. We introduce the rotated frame $\left(G, \mathbf{e}_{1}^{\mathbf{r}}, \mathbf{e}_{2}^{\mathbf{r}}, \mathbf{e}_{3}^{\mathbf{r}}\right)$, following the cross-section plane, in which the coordinates of the material point $M$ are denoted by $y\left(s_{1}, s_{2}\right)$ and $z\left(s_{1}, s_{2}\right)$. We then obtain:

$$
\left\{\begin{array}{l}
\mathbf{O G}=\left(s_{1}+u_{1}\left(s_{1}\right)\right) \mathbf{e}_{1}+u_{3}\left(s_{1}\right) \mathbf{e}_{3} \\
\mathbf{G M}=y\left(s_{1}, s_{2}\right) \mathbf{e}_{2}^{\mathbf{r}}+z\left(s_{1}, s_{2}\right) \mathbf{e}_{3}^{\mathbf{r}}
\end{array}\right.
$$

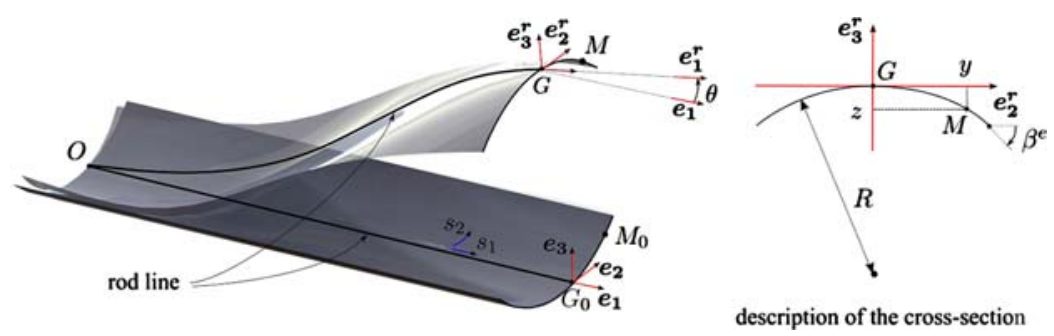

Figure 2. Description of the tape spring. 
According to the assumption (iii), the inextensible cross-section curve can be regarded as an elastica. Introducing the angle $\beta\left(s_{1}, s_{2}\right)$ between the tangent to the cross-section curve and vector $\mathbf{e}_{2}^{\mathbf{r}}$, we have:

$$
\left\{\begin{array}{l}
y_{, 2}=\cos (\beta), \\
z_{, 2}=\sin (\beta) .
\end{array}\right.
$$

Moreover, the cross-section curve is assumed to remain circular. The angle $\beta$ is then linear with respect to $s_{2}$ and the cross-section curve is entirely defined by the angle $\beta^{e}\left(s_{1}\right)$ between the tangent at $s_{2}=a / 2$ and the vector $\mathbf{e}_{2}^{\mathbf{r}}$ (see Figure 2):

$$
\left\{\begin{array}{l}
y\left(s_{1}, s_{2}\right)=\frac{a}{2 \beta^{e}\left(s_{1}\right)} \sin \left(2 \beta^{e}\left(s_{1}\right) \frac{s_{2}}{a}\right), \\
z\left(s_{1}, s_{2}\right)=\frac{a}{2 \beta^{e}\left(s_{1}\right)}\left(1-\cos \left(2 \beta^{e}\left(s_{1}\right) \frac{s_{2}}{a}\right)\right) .
\end{array}\right.
$$

Finally, the tape spring kinematics is described by only four kinematic parameters attached to the rod line:

- the translations $u_{1}\left(s_{1}\right)$ and $u_{3}\left(s_{1}\right)$ of the cross-section;

- the rotation $\theta\left(s_{1}\right)$ of the cross-section around $\mathbf{e}_{2}$ and

- and the angle $\beta^{e}\left(s_{1}\right)$ characterising the shape of the cross-section.

We must notice here that the displacements $u_{1}\left(s_{1}\right)$ and $u_{3}\left(s_{1}\right)$ and the rotation $\theta\left(s_{1}\right)$ are linked by the assumption (iv), that leads to:

$$
\begin{gathered}
C=\mathbf{e}_{\mathbf{3}}^{\mathbf{r}} \cdot \frac{\mathbf{O G}_{\mathbf{1}}}{\left\|\mathbf{O G}_{, \mathbf{1}}\right\|}=\sin (\theta) \frac{1+u_{1,1}}{j^{r}}+\cos (\theta) \frac{u_{3,1}}{j^{r}}=0 \quad \text { with } \\
j^{r}=\left\|\mathbf{O G}_{, \mathbf{1}}\right\|=\sqrt{\left(1+u_{1,1}\right)^{2}+\left(u_{3,1}\right)^{2}} .
\end{gathered}
$$

The novelty of the model resides in the inextensibility assumption of the cross-section curve. It allows to use an elastica kinematics and leads to describe the large change of the cross-section shape with very few parameters, only one here. The idea underlying this assumption is that the tape spring wants to decrease the strain energy by creating some folds, where the cross-section becomes flat to decrease the bending inertia. Due to the large relative displacements inside the cross-section, an inextensibility assumption is sufficient to obtain the overall shape of the cross-section and to efficiently estimate the bending inertia.

\subsection{Strains measures and strain energy}

The tape spring is first considered as a thin shell undergoing membrane and bending strains $e_{\alpha \beta}$ and $k_{\alpha \beta}$ defined, respectively, by the Green-Lagrange measure and the difference between the initial and actual curvature tensors. The tape spring is submitted to large displacements but the membrane strains remain small in practice; since the thickness is very small compared to the two other dimensions $a$ and $L$ of the shell, local and global buckling will prevent large strains to occur. As shown in Guinot et al. (2012), the small membrane strains assumption leads to following simplified expressions: 


$$
\left\{\begin{array} { l } 
{ e _ { 1 1 } = e ^ { r } + z k ^ { r } + e ^ { s } , } \\
{ k _ { 1 1 } = - k ^ { r } \operatorname { c o s } \beta + k _ { 1 1 } ^ { s } , } \\
{ k _ { 2 2 } = k _ { 2 2 } ^ { s } , } \\
{ k _ { 1 2 } = k _ { 1 2 } ^ { s } , }
\end{array} \quad \text { with } \quad \left\{\begin{array}{l}
e^{r}=u_{1,1}+\frac{1}{2}\left(u_{1,1}^{2}+u_{3,1}^{2}\right), \\
k^{r}=\theta_{, 1}, \\
e^{s}=\frac{1}{2}\left(y_{, 1}^{2}+z_{, 1}^{2}\right), \\
k_{11}^{s}=z_{, 11} \cos \beta-y_{, 11} \sin \beta, \\
k_{22}^{s}=\beta_{, 2}-\beta_{0,2}, \\
k_{12}^{s}=\beta_{, 1},
\end{array}\right.\right.
$$

where $\beta_{0}$ is the value of the angle field $\beta$ in the undeformed initial configuration. This Equation (6) enlightens the strains induced by the global rod kinematics (variables with the superscript $r$ ) and those induced by the deformation of the cross-section curve (superscript $s$ ). We recognise in $e^{r}$ and $k^{r}$, the classical expressions of the usual tensile strain and the bending curvature of a rod in the framework of large displacements and large rotations. We can remark that the strains $e^{s}$ and $k_{\alpha \beta}^{s}$ only depend on the angle $\beta$ and vanish if the cross-section remains undeformed. The strains $e_{\alpha \beta}$ and $k_{\alpha \beta}$ are associated with the membrane stresses and bending moments $N_{\alpha \beta}$ and $M_{\alpha \beta}$ in the strain energy. Since the shell width $a$ is small compared to the tape spring length $L$, we suppose that $N_{22}=N_{12}=0$ according to classical beam theory assumptions and we have:

$$
U_{e}=\int_{0}^{L} \int_{-a / 2}^{a / 2} \frac{1}{2}\left(e_{11} N_{11}+k_{\alpha \beta} M_{\alpha \beta}\right) \mathrm{d} s_{2} \mathrm{~d} s_{1} .
$$

Moreover, the shell is considered elastic and isotropic. The constitutive equations are then expressed by:

$$
N_{11}=A e_{11} \text { and }\left\{\begin{array}{l}
M_{11}=D_{11} k_{11}+D_{12} k_{22}, \\
M_{22}=D_{12} k_{11}+D_{22} k_{22} \\
M_{12}=D_{33}\left(2 k_{12}\right),
\end{array}\right.
$$

with

$$
A=E h, D_{11}=D_{22}=\frac{E h^{3}}{12\left(1-v^{2}\right)}, D_{12}=v D_{11}, D_{33}=\frac{E h^{3}}{24(1+v)},
$$

where $h$ is the shell thickness. $E$ and $v$ are the Young's modulus and the Poisson's ratio, respectively. Using the constitutive equations (8) and the expressions (6) for the strains, the strain energy (7) can be written, after integration over the cross-section as:

$$
U_{e}=\int_{0}^{L}\left(u_{e}^{r}+u_{e}^{s}+u_{e}^{r s}\right) \mathrm{d} s_{1}
$$

in which $u_{e}^{r}$, $u_{e}^{s}$ and $u_{e}^{r s}$ are the three terms that define the strain energy density of the rod model: 


$$
\left\{\begin{aligned}
u_{e}^{r}= & \frac{1}{2}\left(A a\left(e^{r}\right)^{2}+\left(A \overline{z^{2}}+D_{11} \overline{\cos ^{2}(\beta)}\right)\left(k^{r}\right)^{2}+2 A \bar{z} e^{r} k^{r}\right), \\
u_{e}^{s}= & \frac{1}{2}\left(A \overline{\left(e^{s}\right)^{2}}+D_{11} \overline{\left(k_{11}^{s}\right)^{2}}+D_{22} \overline{\left(k_{22}^{s}\right)^{2}}\right. \\
& \left.+2 D_{12} \overline{k_{11}^{s} k_{22}^{s}}+4 D_{33} \overline{\left(k_{12}^{s}\right)^{2}}\right) \\
u_{e}^{r s}= & A e^{r} \overline{e^{s}}+A k^{r} \overline{z e^{s}}-k^{r}\left(D_{11} \overline{\cos (\beta) k_{11}^{s}}+D_{12} \overline{\cos (\beta) k_{22}^{s}}\right),
\end{aligned}\right.
$$

where the overline denotes an integration over $s_{2}: \bar{X}\left(s_{1}\right)=\int_{-a / 2}^{a / 2} X\left(s_{1}, s_{2}\right) \mathrm{d} s_{2}$. With the kinematics presented in the previous section, all these integrals can be analytically derived (Guinot et al., 2012), leading to a closed-form expression of the strain energy density depending on the kinematic parameters and the initial characteristics of the tape spring (geometrical and material properties). The first term $u_{e}^{r}$ corresponds to the classical strain energy of a rod with a coupling between axial stretching and bending which appears because the rod line does not pass through the cross-section centroid. The second term $u_{e}^{s}$ only depends on the variable $\beta$ and represents the strain energy due to the variation of the cross-section shape, independently of the overall rod behaviour. The last term $u_{e}^{r s}$ induces a coupling between the overall rod behaviour and the deformation of the cross-section.

\section{Numerical implementation}

The weak form of the problem is needed to use the finite element method. This weak formulation can be obtained by the differentiation of the potential energy with respect to the kinematic parameters $u_{1}\left(s_{1}\right), u_{3}\left(s_{1}\right), \theta\left(s_{1}\right)$ and $\beta^{e}\left(s_{1}\right)$. Let us remind that the first three parameters are constrained by Equation (5) that ensures the orthogonality of the cross-section plane with respect to the rod line. Introducing a Lagrange multiplier $\lambda\left(s_{1}\right)$ associated with the constraint $C\left(s_{1}\right)=0$, we must proceed to the differentiation of the following functional:

$$
\mathcal{P}\left(u_{1}, u_{3}, \theta, \beta^{e}, \lambda\right)=U^{e}-W_{\mathrm{ext}}+\int_{0}^{L} \lambda C \mathrm{~d} s_{1},
$$

where $W_{\text {ext }}$ is the work of external forces, depending on the loading conditions. The model has been implemented in the finite element software COMSOL (COMSOL AB, 2008) that handles the formal differentiation of the potential energy. The aim here is to study the relevance of the model, and not to develop a robust and fast numerical tool. In this way, the finite element software COMSOL allows to overcome the required numerical developments because the expressions (11) can be used as such to define the strain energy density of the rod.

The loading is only applied at the end sections where the following boundary conditions (or their dual force) can be prescribed:

- the translations $u_{1}$ and $u_{3}$ of the cross-section;

- the rotation $\theta$ of the cross-section;

- the angle $\beta^{e}$ that defines the transverse curvature of the cross-section and

- the first derivative $\beta_{, 1}^{e}$ of this angle that prescribes the local rotation at each point of the cross-section curve (clamped end section for example). 
In the following, the loading is defined in terms of prescribed displacements or rotation at ends. The default time-dependent solver (backward differentiation formula: BDF solver) of COMSOL is used because it allows to introduce small viscous terms in the weak formulation to face with the snap-through phenomena. Finally, the discretisation of the rod line is performed with one-dimensional Hermite quintic finite elements to take into account the highorder derivatives that appear in the model for the opening angle $\beta^{e}$.

\section{Numerical applications}

\subsection{Introduction}

The geometrical and material properties of the tape spring under consideration are given in Table 1. In the following, several scenarios of buckling are studied with several kinds of boundary conditions. It is shown that it always leads to the creation of one or more folds that can move along the tape, disappear, merge or split. In the following figures, the $3 \mathrm{D}$ deformed shapes of the tape spring are reconstructed, thanks to the results of the $1 \mathrm{D}$ rod model and the kinematics exposed in Section 2.1. The colour plot of $\beta$ are superimposed on the deformed shapes to give information on the local $z$-coordinate in the local cross-section frame. The dark blue regions correspond to areas where the cross-section is completely flattened; $z$ increases from dark blue $(z=0)$ to red. The static analyses of buckling are performed under the assumption that the tape spring remains symmetric with respect to the plane $\left(O, \mathbf{e}_{\mathbf{1}}, \mathbf{e}_{\mathbf{3}}\right)$. An extension of the model to 3D motions and out-of-plane buckling analysis constitutes an ongoing work.

\subsection{Buckling of the cross-section under pure overall bending}

A pure overall bending is obtained with prescribed opposite rotations $\theta$ at the end sections. The other boundary conditions are given by: $u_{1}=u_{3}=0$ and $\beta^{e}=\beta_{0}^{e}$ at one end and $u_{3}=0, \beta^{e}=\beta_{0}^{e}$ and $u_{1}$ free at the other end. Figure 4 (left) shows that at the beginning, for small values of the prescribed angle $\theta(L)$, the tape spring behaves as a classical beam (CBT) with non-deformable cross-sections; the response is linear. We then observe $(0.04<\theta(L)<0.12) \mathrm{rad})$ a non-linearity, due to a non-uniform flattening of the cross-sections all along the tape. The maximum flattening is obtained in the middle $s_{1}=\frac{L}{2}$ but there is no localisation (deformed shape 2 in Figure 3). The bending moment reaches a maximum and decreases before a snap-through that occurs for a prescribed rotation of $\theta(L)=0.12 \mathrm{rad}$. The tape switches from a first equilibrium branch to another one that corresponds to the creation of a localised fold (deformed shape 3 in Figure 3). In the fold region, the cross-section is completely flat and the bending inertia is much smaller that the initial one. It leads to an important loss of the tape bending strength. When the prescribed rotation is decreased, we observe other snap-through phenomena. Figure 4 (right) shows the evolution of the strain energy density $u_{e}=u_{e}^{r}+u_{e}^{s}+u_{e}^{r s}$ along the tape with respect to the prescribed rotation $\theta(L)$. This plot clearly illustrates the sudden localisation of the strain energy density. These results are in agreement with the results obtained in Seffen et al. (2000).

Table 1. Geometrical and material properties of the tape spring.

\begin{tabular}{cccccc}
\hline Length $L$ & Width $a$ & Thickness $h$ & Initial angle $\beta_{0}^{e}$ & Young's modulus $E$ & Poisson's ratio $v$ \\
\hline $1170 \mathrm{~mm}$ & $60 \mathrm{~mm}$ & $0.15 \mathrm{~mm}$ & $0.6 \mathrm{rad}$ & $210,000 \mathrm{MPa}$ & 0.3 \\
\hline
\end{tabular}




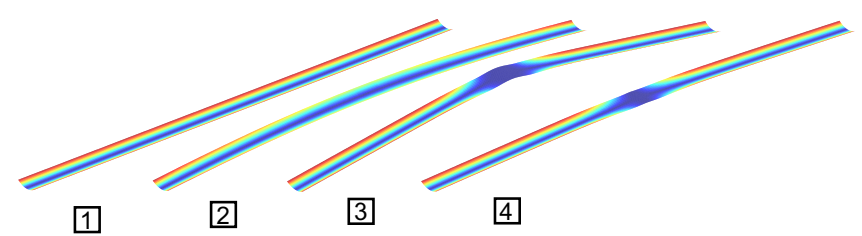

Figure 3. Creation of a fold under pure overall bending. Deformed shapes with superimposed colour plots of the angle $\beta$, indicating the level of flattening of the cross-section.
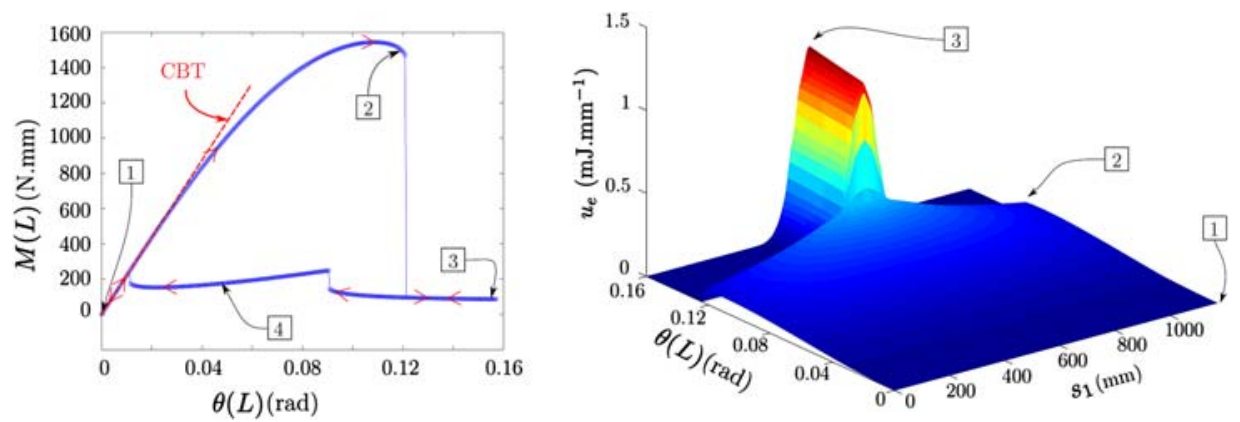

Figure 4. Reaction moment with respect to the prescribed rotation (left). Strain energy density along the tape with respect to the prescribed rotation (right).

\subsection{Buckling under compression}

A pure compressive load is prescribed at the end sections of the tape. When this load is applied at the centroid of the initial cross-section, the tape remains straight, corresponding to the trivial solution $\left(u_{3}\left(s_{1}\right)=\theta\left(s_{1}\right)=\beta^{e}\left(s_{1}\right)=0\right)$. On this trivial solution branch, the first bifurcation branch is asymmetric. In the following, loading imperfections are introduced to investigate the buckling and post-buckling behaviour under an overall compression. In particular, we study the compressive reaction force with respect to the prescribed compressive axial displacement. Boundary conditions are known to play an important role in the buckling behaviour of beams (Bazant \& Cedolin, 1991; Nguyen, 2000; Timoshenko \& Gere, 1961). For tape springs, it is shown hereafter that they have a direct impact on the number and localisation of the folds that can appear.

\subsubsection{Free rotations at ends}

In this first case, the rotation $\theta$ is free and the flexural displacement $u_{3}$ is fixed at each end. The compressive load is applied by imposing a displacement $u_{1}$ at one end $\left(s_{1}=L\right)$ whereas the other is fixed. Moreover, the cross-section shape is fixed at each end $\left(\beta^{e}=\beta_{0}^{e}\right)$. The imperfection consists of a small flexural force $F_{3}=10^{-4} \mathrm{~N}$ applied at the middle of the tape, leading to a small opposite bending that preserves the symmetry with respect to the plane $(O$, $\mathbf{e}_{1}, \mathbf{e}_{3}$ ). Figure 5 illustrates the overall behaviour of the tape spring. In the first part, the response curve follows (up to the state 1) the usual scenario of a perturbed bifurcation branch, getting closed to the trivial perfect compressive solution first and then turning sharply to follow the bifurcated branch with a decreasing strength. We observe then a snap-through to another branch, accompanied by the creation of a fold. This branch is characterised by a con- 

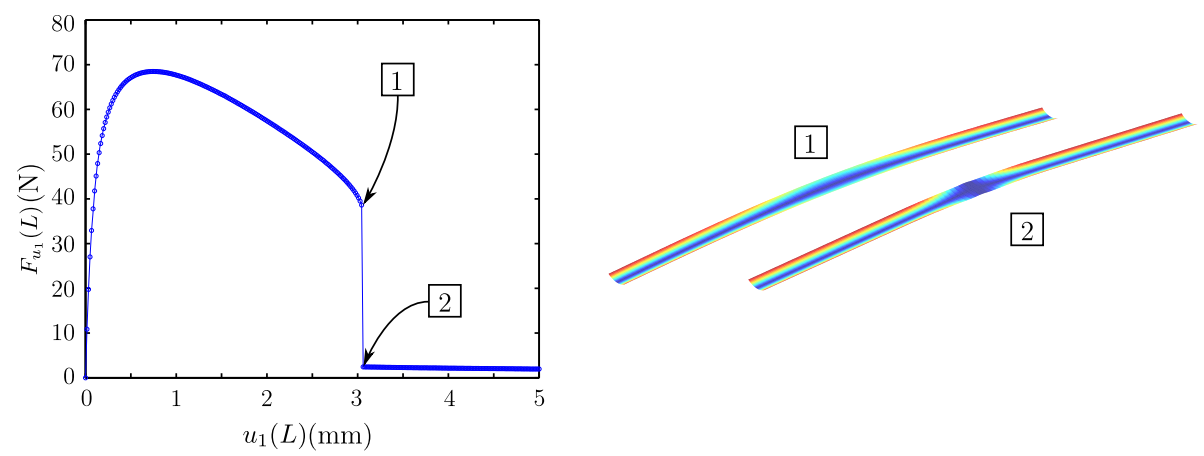

Figure 5. Buckling under compression with free rotations at ends. The deformed shapes with the superimposed colour plot of $\beta$ illustrate the creation of the fold (right). The plot of the compressive reaction force with respect to the axial displacement at end shows the sudden snap-through (left).
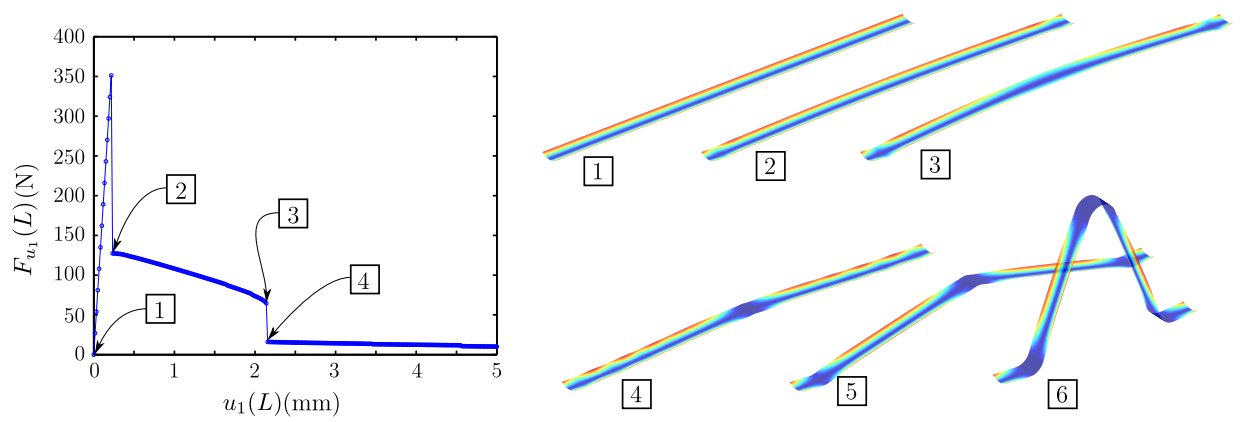

Figure 6. Buckling under compression with fixed rotations at ends. The deformed shapes with the superimposed colour plot of $\beta$ illustrate the creation of the folds (right). The plot of the compressive reaction force with respect to the axial displacement at end shows the snap-throughs (left).

stant reaction force that does not depend on the prescribed displacement at the end section. This snap-through is similar in nature to the case presented in Section 4.2. This is again the overall bending that leads to the flattening of the cross-section in a localised region of the tape. But in the present case, the overall bending occurs during the post-buckling.

\subsubsection{Fixed rotations at ends}

Contrary to the previous case, the rotation $\theta$ is fixed at each end of the tape. Since the crosssection is not allowed to rotate, we observe at the very beginning a snap-through with the creation of two localised regions of flattening near the ends, where the overall bending moment generated by the flexural displacement in the middle is maximum (see deformed shape 2 in Figure 6). These partial foldings are followed by an overall bending with a distributed flattening all along the tape with a maximum that takes place in the middle (see the evolution of colour plot of $\beta$ on the deformed shape 3 ). This flattening increases, leading to a second snap-through to another equilibrium branch for which a significant fold takes place in the middle and the flattening near the ends are somewhat released (deformed shape 4). Furthermore, we observe some oscillations on the colour plot of $\beta$ in this deformed state, indicating that local buckling occurs due to localised compressive loads at the ends of the cross-section curve. Increasing the prescribed displacement at end removes these oscillations, 

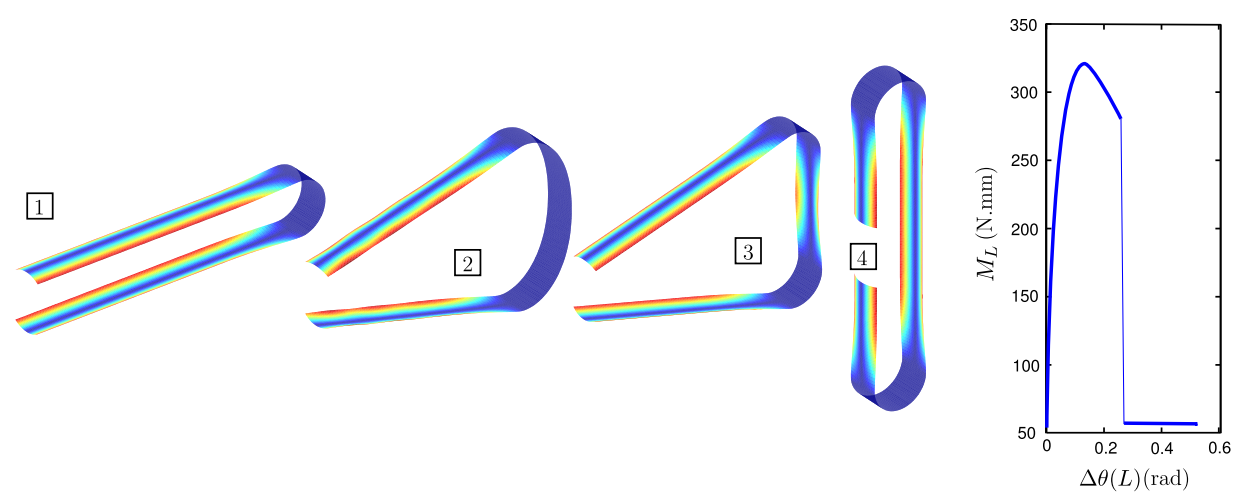

Figure 7. Splitting of a fold into two.

restores the folds near the ends and increases the folding region in the middle (deformed shapes 4 and 5).

The results obtained in these two cases (free and fixed rotations at ends) show that such a structure is highly non-linear and sensitive to imperfections. A linearised buckling analysis is then unsuitable for predicting the buckling behaviour of tape springs.

\subsection{Splitting of a fold into two}

The starting state is the deformed shape 1 shown in Figure 7. This state is obtained with the same boundary conditions than those used in Section 4.2, except that the bending is in the opposite sense. The boundary conditions are then modified: the displacements $u_{1}$ and $u_{3}$ are fixed at each end in the deformed shape 1 and opposite increments of rotation $\Delta \theta$ are prescribed to spread the two straight parts of the tape (from 0 to $\pi / 2$ at one end and 0 to $-\pi / 2$ at the other one). At the beginning, the rotation of the straight parts of the tape leads to an extension of the region of flattening in the middle (see deformed shapes 1 and 2 in Figure 7). We also observe some waves on the colour plot of deformed shape 2, indicating localised buckling due to local compression. This intermediate state is unstable and a snap-through occurs between the states 2 and 3 that leads to the duplication of the fold which relaxes the local compression. In the region between the two new folds, the cross-section recovers its initial shape. The two new folds have the same properties than the initial single one: the fold regions are cylindrical with a completely flat cross-section and a longitudinal curvature equal to the initial transverse curvature of the cross-section (Guinot et al., 2012). Increasing the increment of rotation moves the two folds away from each other.

\section{Conclusions}

In this work, we have recalled the basics of the rod model with flexible cross-sections proposed in Guinot et al. (2012). This 1D planar rod model involves only four parameters and is suitable to simulate the behaviour of tape springs. It is shown that this approach is able to handle complex scenarios of buckling, leading to the creation, duplication and migration of folds along the tape. Compared to shell models, this extended rod model is easier to handle and naturally involves some mechanical quantities of interest such as the bending inertia, con- 
trolled by the kinematic parameter $\beta^{e}$ that governs the cross-section shape. This approach enables to describe but also to understand the main phenomena that control the behaviour of tape springs. The COMSOL software and its time dependent solver (BDF solver) was convenient here for testing and evaluating the model without writing lines of code. However, it does not allow to get the full bifurcation diagram and this may limit the understanding of the complete behaviour. We are currently working on the use of a path following algorithm (asymptotic numerical method) to obtain the full equilibrium diagram with stable and unstable branches of solutions, together with the definition of a suitable 1-D finite element, for an efficient and reliable numerical implementation of the proposed tape-spring model.

\section{References}

Bazant, Z.P., \& Cedolin, L. (1991). Stability of structures: Elastic, inelastic, fracture, and damage theories. New York, NY: Oxford University Press.

Comsol (2008). Comsol Multiphysics User's Guide, Comsol AB, Stockholm, http://www.comsol.com.

Gonçalves, R., Ritto-Corrêa, M., \& Camotim, D. (2010). A large displacement and finite rotation thinwalled beam formulation including cross-section deformation. Computer Methods in Applied Mechanics and Engineering, 199(23-24), 1627-1643.

Guinot, F., Bourgeois, S., Cochelin, B., \& Blanchard, L. (2012). A planar rod model with flexible thinwalled cross-sections: Application to the folding of tape springs. International Journal of Solids and Structures, 49(1), 73-86.

Hoffait, S., Bruls, O., Granville, D., Cugnon, F., \& Kerschen, G. (2009). Dynamic analysis of the selflocking phenomenon in tape-spring hinges. Acta Astronautica, 66(7-8), 1125-1132.

Nguyen, Q.S. (2000). Stability and nonlinear solid mechanics. Chichester: Willey.

Pimenta, P., \& Campello, E. (2003). A fully nonlinear multi-parameter rod model incorporating general cross-sectional in-plane changes and out-of-plane warping. Latin American Journal of Solids and Structures, 1(1), 119.

Santer, M., \& Pellegrino, S. (2008). Compliant multistable structural elements. International Journal of Solids and Structures, 45(24), 6190-6204.

Seffen, K. (2001). On the behavior of folded tape-springs. Journal of Applied Mechanics - Transactions of the ASME, 68(3), 369-375.

Seffen, K., \& Pellegrino, S. (1999). Deployment dynamics of tape springs. Proceedings of the Royal Society London A, 455, 1003-1048.

Seffen, K., You, Z., \& Pellegrino, S. (2000). Folding and deployment of curved tape springs. International Journal of Mechanical Sciences, 42(10), 2055-2073.

Simo, J., \& Vu-Quoc, L. (1991). A geometrically-exact rod model incorporating shear and torsion-warping deformation. International Journal of Solids and Structures, 27(3), 371-393.

Soykasap, O. (2007). Analysis of tape spring hinges. International Journal of Mechanical Sciences, 49 (7), 853-860.

Timoshenko, S.P., \& Gere, J.M. (1961). Theory of elastic stability. New-York, NY: Mc Graw-Hill.

Vlassov, B. (1962). Pièces longues en voiles minces (Long thin-walled beams). Paris: Eyrolles.

Walker, S., \& Aglietti, G. (2007). A study of tape spring fold curvature for space deployable structures. Proceedings of the Institution of Mechanical Engineers Part G - Journal of Aerospace Engineering, 221(G3), 313-325.

Zivkovic, M., Kojic, M., Slavkovic, R., \& Grujovic, N. (2001). A general beam finite element with deformable cross-section. Computer Methods in Applied Mechanics and Engineering, 190(20-21), 2651-2680. 